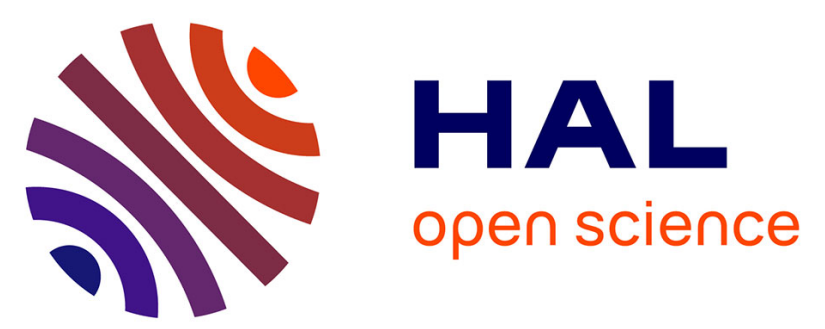

\title{
Continuous chemical operations and modifications on magnetic $\gamma$-Fe2O3 nanoparticles confined in nanoliter droplets for the assembly of fluorescent and magnetic $\mathrm{SiO} 2 @ \gamma-\mathrm{Fe} 2 \mathrm{O} 3$
}

D Ferraro, Y Lin, B Teste, Damien Talbot, L Malaquin, S Descroix, Ali Abou

\author{
Abou-Hassan
}

\section{To cite this version:}

D Ferraro, Y Lin, B Teste, Damien Talbot, L Malaquin, et al.. Continuous chemical operations and modifications on magnetic $\gamma$-Fe2O3 nanoparticles confined in nanoliter droplets for the assembly of fluorescent and magnetic SiO2@ $\gamma$-Fe2O3 . Chemical Communications, 2015, 51 (95), pp.16904-16907. 10.1039/C5CC07044A . hal-01219164

\section{HAL Id: hal-01219164 \\ https://hal.sorbonne-universite.fr/hal-01219164}

Submitted on 22 Oct 2015

HAL is a multi-disciplinary open access archive for the deposit and dissemination of scientific research documents, whether they are published or not. The documents may come from teaching and research institutions in France or abroad, or from public or private research centers.
L'archive ouverte pluridisciplinaire HAL, est destinée au dépôt et à la diffusion de documents scientifiques de niveau recherche, publiés ou non, émanant des établissements d'enseignement et de recherche français ou étrangers, des laboratoires publics ou privés. 


\title{
Continuous chemical operations and modifications on magnetic $\mathrm{Y}-\mathrm{Fe}_{2} \mathrm{O}_{3}$ nanoparticles confined in nanoliter droplets for the assembly of fluorescent and magnetic $\mathrm{SiO}_{2} @ \gamma-\mathrm{Fe}_{2} \mathrm{O}_{3}$
}

\author{
D. Ferraro, ${ }^{a}$ Y.Lin, ${ }^{a, b}$ B. Teste, ${ }^{a}$ D. Talbot, ${ }^{b}$ L. Malaquin, ${ }^{a}$ S. Descroix, ${ }^{a^{*}}$ and A. Abou-Hassan ${ }^{b^{*}}$
}

\begin{abstract}
We present a microfluidic platform that allows undergoing different chemical operations in a nanoliter droplet starting from colloidal suspension of magnetic iron oxide $\left(\gamma-\mathrm{Fe}_{2} \mathrm{O}_{3}\right)$ nanoparticles "NPs" (ferrofluid). These operations include: mixing, flocculation, magnetic decantation, colloidal redispersion, washing, surface functionalization, heating and colloidal assembly. To prove the platform capabilities, we produced fluorescent and magnetic nanoassemblies composed of fluorescent silica and magnetic NPs.
\end{abstract}

Due to their specific properties rising from their small dimensions, nanoparticles (NPs) are used in various applications such as catalysis, optics, electronics and biomedical diagnosis or nanomedicine. ${ }^{1,2}$ To extend even more the range of properties and applications of NPs, several nanomaterials can be combined into one single nanostructure to form a multifunctional nanohybrid. This can be accomplished by controlling the assembly of smaller nanostructures (building blocks) of various shapes and compositions to create complex and single nanoscale object, having different properties provided by each of the different nanomaterials. ${ }^{3}$

In the context of nanoparticle synthesis and assembly, different microfluidic strategies had already been shown with a high level of synthesis control enabling the production of high quality nanostructures with single or multi-functionalities. ${ }^{4,5}$ Particularly, in materials science, droplet microfluidics has been identified as a powerful tool to control chemical reactions through their compartmentalisation inside individual droplets, where each droplet acts as a micro-reactor allowing reaction parallelisation, reduction of reagents volume and accurate mixing associated to an enhancement of mass and heat transfer compared with bulk reactions. ${ }^{6}$ Moreover, it is also possible to manipulate individually each droplet to perform complex operations such as splitting, merging, sorting that allow to combine different chemical

\footnotetext{
a. Laboratoire Physicochimie Curie, CNRS/UMR 168, 11 rue Pierre et Marie Curie, Paris, France.

${ }^{b}$ Sorbonne Universités, UPMC Univ. Paris 06, Laboratoire de PHysico-chimie des Electrolytes et NanosystèmesInterfaciauX (PHENIX), case 51, 4 place Jussieu, F75252 Paris Cedex 05 (France).

Electronic Supplementary Information (ESI) available: [microfluidic drroplets heater, TEM and magnetic characterisations of magnetic nanoparticles, phase diagram for trapping magnetic nanoparticles, TEM images of the building blocks, preparation of TEM grids and Movies captions]. See DOI: 10.1039/x0xx00000x
}

reactions. ${ }^{7,8}$ Despite several convincing reports on the use of microfluidics for the elaboration of nanomaterials through chemical synthesis or self-assembly, ${ }^{9-11}$ there is still rooms for high impact studies relying on technology for complex workflow implementation at microfluidic scale. In particular, manipulations of magnetic nanoparticles in microfluidic systems are still limited to single step reactions, ${ }^{12}$ or multistep continuous modifications, ${ }^{13}$ when operations as extraction, washing or NP functionalisation are not required or performed out from the microfluidic devices by conventional approaches. This is mainly due to their nanoscale dimension which renders difficult their handling in confined condition, where low amount of NPs, or in other words low amount of paramagnetic materials, results in low magnetic force. In these states typical macroscale protocols cannot be directly exploited. Herein we provide for the first time an automated microfluidic platform capable of manipulating nanoliter droplets and performing multistep and automated sequences of operations on magnetic NPs. These operations include screening of colloidal interactions magnetic decantation, colloidal redispersion, washing, surface functionalization, heating and colloidal assembly. In order to illustrate the potential of this microfluidic technology we report the elaboration of fluorescent and magnetic nanoassemblies from fluorescent silica and magnetic NPs as building blocks. Nanohybrid structures with dual functionalities are very promising and have proven their potential in different biomedical contexts such as drug delivery, magnetic resonance imaging (MRI) and hyperthermia cancer treatment. ${ }^{2,14}$

Figure 1a summarises the fully automated droplet-based microfluidic platform, which is able to generate droplets, control the liquid handling, the magnetic actuation, as well as the droplet temperature. It is composed of a syringe pump equipped with $250 \mu \mathrm{L}$ syringe (by SGE) and coupled with a pipetting robot (Nemesys and Rotaxys, by Cetoni $\mathrm{GmbH}$ ) that allows the generation of trains of droplets starting from solutions collected in a standard microtiter plate. A PTFE capillary (inside/outside diameter of 300 $\mu \mathrm{m} / 600 \mu \mathrm{m}$, by Sigma Aldrich) is used as a pipetting needle to aspirate alternatively the different reagent solutions and fluorinated oil (FC-40 by $3 \mathrm{M}$ ) that is used as continuous phase. This module, which is inspired by the one presented by Chabert et $a l^{15}$, offers the possibility to generate a deterministic and confined train of droplets in a range between 50 and $300 \mathrm{~nL}$ (see example in Figure $1 b$ ) with an error in volume of about $2 \%$. In order to generate stable 


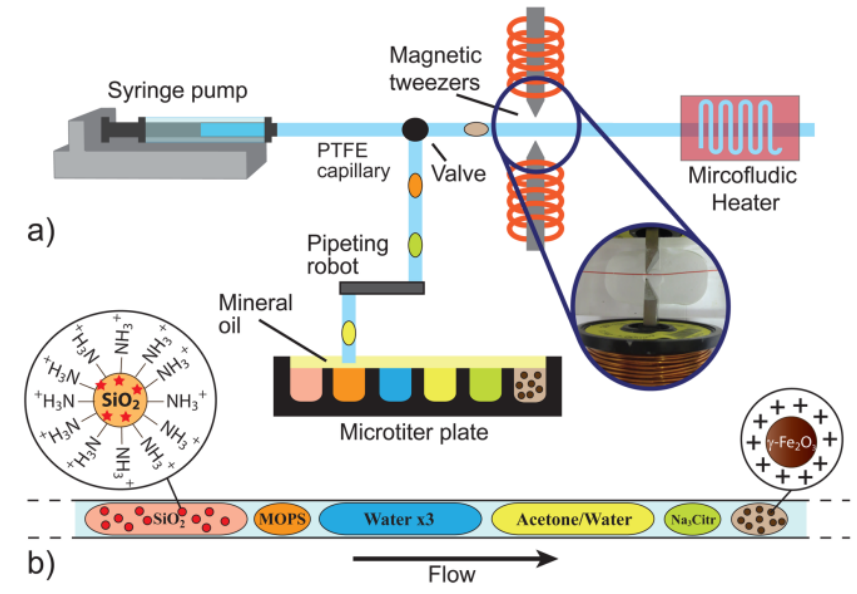

Figure 1. a) Microfluidic setup for the generation of deterministic trains of droplets. Syringe pump synchronised with a pipetting robot generates the droplet from a microtiter plate, sucking water phase solutions and oil alternatively. These droplets are then driven between the magnetic tweezers (picture in the inset) for ferrofluid manipulation and the homemade heater that can be easily tuned at the desired temperature. b) Train of droplets for nanoassembling production: 1) $50 \mathrm{~nL}$ of $\gamma-\mathrm{Fe}_{2} \mathrm{O}_{3}$ solution, 2) $50 \mathrm{~nL}$ of sodium citrate solution, 3) $200 \mathrm{~nL}$ of acetone/water solution, 4) 2 drops of $200 \mathrm{~nL}$ of milliQ water, 5) $50 \mathrm{~nL}$ of $3-(\mathrm{N}$ Morpholino)propanesulfonic acid (MOPS) and 6) $200 \mathrm{~nL}$ of silica fluorescent NPs.

droplets, FC-40 is mixed with $2 \%(\mathrm{w} / \mathrm{w})$ of a fluorinated surfactant $(1 \mathrm{H}, 1 \mathrm{H}, 2 \mathrm{H}, 2 \mathrm{H}$-perfluorodecan-1-ol, by Fluorochem). Additionally, the microtiter plate is entirely covered with a small layer (about $2 \mathrm{~mm}$ ) of mineral light oil (Sigma Aldrich) to avoid the reagent evaporation. Since the syringe pump can work in both injections and withdrawal modes, droplets can be moved forward and backward in the capillary. This capability will be exploited for inducing a recirculation inside the droplet, which helps the internal mixing. ${ }^{16}$ Once the train of droplets is generated, droplets can be driven between the magnetic tweezers to achieve the manipulation of the magnetic NPs and through a homemade heater (see ESI, Figure S1), which allows the temperature control of the droplet. The magnetic tweezers consist of two sharp magnetic tips (AFK502 Imphy Alloys) facing each other across a capillary where the droplets are flowing. Each tip of the tweezers can be activated by a magnetic coil. Therefore it is possible to switch ON and OFF the magnetic field simply by controlling the coil current. The power of this technology had already been validated for extraction or agglutination of magnetic micrometric beads for protein detection, ${ }^{17,18}$ however the manipulation of colloidal magnetic NPs has never been demonstrated before in droplet microfluidics.

The challenge of the manipulation of NPs compared to micrometric beads in microfluidic droplets could be explained considering the forces acting in the system. For example, it has been shown that to extract micrometric particles from a droplet, the magnetic field allows to collect them in a cluster that can be extracted if the magnetic force is higher than the capillary force ${ }^{17}$ (see Movie 1 , ESI). After that, the cluster of beads can be merged and released in the following droplet. However, in case of ferrofluid the small dimension of the particles favours the dispersion forces relative to
Brownian motion. In particular, in this case we noticed that the entire aqueous droplet is trapped, but no cluster formation and extraction are observed (see Movie 2, ESI). In fact, NPs experience two opposite forces: the magnetic force which tends to aggregate them and the colloidal force which keeps the suspension stable, resulting in a metastable system where the droplet is trapped at the magnetic tweezers (see paragraph 2 in ESI). Nevertheless, it is interesting to notice that even if it does not permit particle extraction, this effect can be advantageously exploited for merging two following droplets.

Size sorted cationic ferrofluids of maghemite $\left(\nu-\mathrm{Fe}_{2} \mathrm{O}_{3}\right)$ NPs suspended in nitric acid $(\mathrm{pH}=2)$, with different diameters $\left(d_{0}\right.$ between 5-9nm; see ESI, Figure S3 for more details) were synthesised according to our previous described methods. ${ }^{19,20} \mathrm{~A}$ phase diagram describing the efficiency of ferrofluid droplet trapping effect was built to report the influence of NPs diameter and iron concentration ([Fe]) at constant oil flow rate $\left(Q=0.02 \mu \mathrm{L} \cdot \mathrm{s}^{-1}\right)$ (see ESI, Figure S4). $Q$ was chosen in order to be able to trap also the droplet of ferrofluid with smaller NPs at lower concentration of iron. Since the magnetic force is proportional to the quantity of magnetic material, as expected bigger and higher concentrated NPs are more suitable for the droplet trapping. For example fixing $[\mathrm{Fe}]=0.3 \mathrm{~mol} \cdot \mathrm{L}^{-1}$, only droplet containing NPs having $d_{0} \geq 7 \mathrm{~nm}$ can be trapped.

In order to perform complex ferrofluid manipulation, it is necessary to be able to extract the NPs from the ferrofluid. This can be achieved increasing the magnetic field to overcome the colloidal force and generate a cluster, as described for micrometric particles, or destabilizing the colloidal suspension. Considering the difficulties to generate high magnetic fields using electromagnets, the latter solution was found to be simpler and more straightforward to implement experimentally. In particular, this was achieved by merging the droplet of ferrofluid with a droplet of acetone, which increases the solvent permittivity leading to NPs flocculation. At this point, NPs are not stable in the solutions, they aggregate quickly and accumulate at the tweezers forming a cluster that can be extracted from the initial droplet (see Movie 3, ESI) and released in any following droplet or washed with subsequent droplets.

Based on these validations, we demonstrated the potential of the platform to perform series of complex synthesis workflows. In particular we focused on the elaboration of magnetic and fluorescent nanoassemblies from maghemite $\left(\gamma-\mathrm{Fe}_{2} \mathrm{O}_{3}\right)$ and silica NPs as building blocks. We have selected $\gamma-\mathrm{Fe}_{2} \mathrm{O}_{3} \mathrm{NPs}$ of $d_{0} \approx 9 \pm 0,23$ $\mathrm{nm}$ as the magnetic building block since NPs with this size are easier to manipulate using our tweezers and have proven their efficiency in different applications related to nanomedicine. ${ }^{21}$ The other building block is amine functionalised fluorescent silica NPs (RITC$\left.\mathrm{SiO}_{2}\right)(\mathrm{d} \approx 200 \pm 4 \mathrm{~nm}$; ESI Figure S5) that have been synthesised according to the modified Stöber method using the Rhodamine B isothiocyanate (RITC, $\lambda_{\text {absorption }}=555 \mathrm{~nm}$ ) as a fluorescent dye. ${ }^{22}$ The amine functionalised silica NPs are stable at $\mathrm{pH}=7$ in $10^{-3} \mathrm{~mol} \cdot \mathrm{L}^{-1}$ of 3-(N-Morpholino)propanesulfonic acid (MOPS) pH buffer and their zeta potential is $\zeta=+35 \mathrm{mV}$.

To build our assembly through electrostatic interaction at $\mathrm{pH}=7$ between the positively charged silica and the maghemite NPs, we 
decided to coat their surface with citrate molecules since maghemite NPs are not stable at this $\mathrm{pH} .{ }^{13}$ The role of this coating is to ensure their colloidal stability due to carboxylate functional groups on their surface, which also guarantee a strong interaction with positively charged silica NPs.

Therefore, the workflow necessary for reaching the final assembly, which is completely performed in droplets, is illustrated in Figure 2a. At first, the train of droplets sketched in Figure $1 \mathrm{~b}$ is generated: 1) $50 \mathrm{~nL}$ droplet of $\gamma-\mathrm{Fe}_{2} \mathrm{O}_{3}$ solution $\left(d_{0} \approx 9 \mathrm{~nm}, \quad[\gamma-\right.$ $\left.\mathrm{Fe}_{2} \mathrm{O}_{3}\right] \approx 8.2 \times 10^{18} \mathrm{NPs} \cdot \mathrm{L}^{-1}$ or $\left.[\mathrm{Fe}] \approx 0.44 \mathrm{~mol} \cdot \mathrm{L}^{-1} ; 2\right) 50 \mathrm{~nL}$ of $0,027 \mathrm{~mol} \cdot \mathrm{L}^{-1}$ trisodium citrate solution giving a molar ratio $\mathrm{Fe} / \mathrm{citrate} \approx 16$; 3 ) $200 \mathrm{~nL}$ of an acetone/water mixture $(50 / 50 \mathrm{v} / \mathrm{v})$; 4) two water droplets of $200 \mathrm{~nL}$ each; 5) $50 \mathrm{~nL}$ of $10^{-3} \mathrm{~mol} / \mathrm{L}$ MOPS $\mathrm{pH}$ buffer solution $(\mathrm{pH}=7)$ and 6) $200 \mathrm{~nL}$ of silica fluorescent NPs $(d \approx 200 \mathrm{~nm}$, [RITC-SiO ${ }_{2}$ ] $\approx 1.88 \times 10^{15} \mathrm{NPs} \cdot \mathrm{L}^{-1}$ ). Based on the trapping mechanism previously described, the droplet containing $\gamma-\mathrm{Fe}_{2} \mathrm{O}_{3}$ is trapped at the tweezers and merged with the following droplet containing trisodium citrate (step 1, Figure $2 \mathrm{~b}$-g) to perform the NPs citration. The addition of trisodium citrate solution induced nanoparticle destabilisation and precipitation in the droplet (see Movie 4 ESI) due to the high ionic strength. To accelerate diffusion and grafting of citrate molecules on the surface of magnetic NPs, the resulting mixture in the droplet was flown in the microfluidic heater introduced above and heated at $60^{\circ} \mathrm{C}$ for 5 minutes (step 2 in Figure 2). After this step, the resulting suspension of $\gamma-\mathrm{Fe}_{2} \mathrm{O}_{3}$ became optically homogeneous indicating uniform dispersion of stabilized citrate functionalised magnetic NPs in the droplet. Since the sodium citrate is in excess, we expected that the reaction efficiency is $100 \%$ and all the NPs resulted functionalized. To remove the excess of citrate molecules, the droplet from step 2 was magnetically trapped again and merged with a droplet containing the mixture of acetone/water, while keeping the magnetic field and the oil flow ON (step 3, Figure 2h-m, Movie 3 ESI). As previously introduced, thanks to the acetone, we could extract the citrated magnetic NPs from the excess of citrate presented in the droplet solution. In the step 4 (figure $2 n-s$ ), the NPs cluster was washed with two water droplets, and finally released in the MOPS solution. During washing, the flocculated NPs through which the water droplets flowed were kept trapped at the tweezers. Figure $2 n-p$ and Movie 5 in ESI show how the cluster becomes larger when the water droplets pass through, evidencing slight redispersion of the NPs. Additionally some $\gamma-\mathrm{Fe}_{2} \mathrm{O}_{3} \mathrm{NPs}$ are lost in the washing droplet; evaluating the grey levels of the Movie 5, we estimate that this quantity is about the $10 \%$ of the total cluster of NPs. Then, when the MOPS solution was mixed with the magnetic NPs (Figure 2q-s and Movie 6 ESI), the resulting droplet was released from the tweezers and flown for 1 minute in the capillary to obtain a better mixing. At this stage, no NPs sedimentation was observed even after 1 hour suggesting the efficient stability of $\mathrm{\gamma}-\mathrm{Fe}_{2} \mathrm{O}_{3}$-citrated NPs in MOPS. Finally, the droplet containing the citrated magnetic NPs dispersed in MOPS was trapped at the tweezers and merged with a droplet loaded with

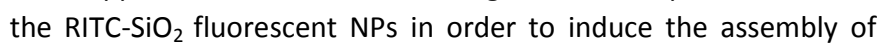
$\mathrm{RITC}-\mathrm{SiO}_{2} @ \mathrm{Y}-\mathrm{Fe}_{2} \mathrm{O}_{3}$ nanohybrids through electrostatic interactions. As presented in the previous case, mixing was done flowing the resulting droplet in the capillary for 1 minute in order to benefit from the internal recirculation flows.
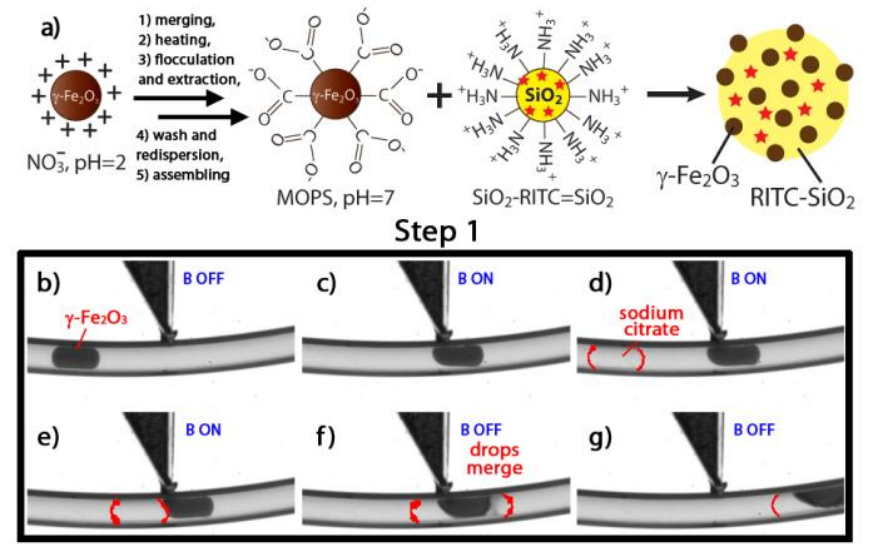

Step 2

$7 \begin{gathered}\text { Heating } \\ 60^{\circ} \mathrm{C}-5 \mathrm{~min}\end{gathered}$

Step 3



Step 4

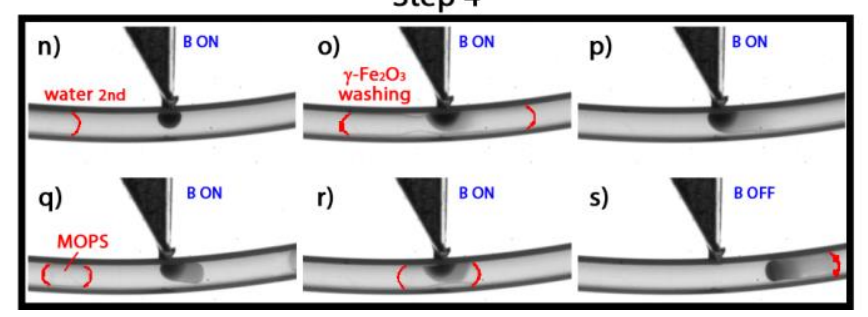

Figure 2. (Top) a) scheme of the sequential reaction and workflows for the elaboration of nanoassemblies starting from the aqueous suspension of ferrofluid at $\mathrm{pH}=2$; (Bottom) Manipulation of $\gamma-\mathrm{Fe}_{2} \mathrm{O}_{3} \mathrm{NPs}$ confined in droplet by magnetic tweezers: b)-g) (Step 1) $\gamma-\mathrm{Fe}_{2} \mathrm{O}_{3}$ droplet is trapped and mixed with a following droplet which in this case contains sodium citrate solution; (Step 2) the resulting droplet is heated for 5 minutes at $60^{\circ} \mathrm{C} ; \mathrm{h}$ )m) (Step 3) destabilizing by acetone/water droplet of the $y-\mathrm{Fe}_{2} \mathrm{O}_{3}$ solution, NPs flocculate and can be extracted; n)-s) (Step 4) 2 water droplets pass through the NPs cluster for washing (in this figure we present only the second water droplet) and then $-\mathrm{Fe}_{2} \mathrm{O}_{3}$ NPs are released in the MOPS droplet. Transparent droplets are highlighted by red lines on the meniscus. The direction of the flow is from the left to the right.

Optical fluorescence microscopy was used to confirm the successful formation of RITC-SiO $@ \mathrm{Y}^{-} \mathrm{Fe}_{2} \mathrm{O}_{3}$ assemblies in the nanodroplets. As shown in Figure $3 a$ when the magnetic field (B) is OFF, the nanoassemblies obtained in the droplet are randomly distributed due to Brownian motion; while in presence of a permanent magnetic field (Figure 3b), the fluorescent homogenized nanoparticles lined up (white arrow) along the magnetic field direction (see also Movie 7, ESI) to form chainlike structures due to the attractive interaction between the magnetic moments. Additionally, applying a magnetic field gradient to the RITC-SiO ${ }_{2} @$ y$\mathrm{Fe}_{2} \mathrm{O}_{3}$ assemblies confined in droplet by a permanent magnet, we observed that they tend to agglomerate close to it (see Movie 8, 




Figure 3. Fluorescent images of the silica nanoparticles coated with iron oxide nanoparticles in the absence (a) and in the presence of the magnetic field (b), oriented as indicated by the white arrow; c-d) TEM images of RITC-SiO $@_{2}-\mathrm{Fe}_{2} \mathrm{O}_{3}$ nanoassemblies.

ESI). These effects support the bifunctional character (magnetic and fluorescent) of the nanoassemblies. The droplet was next flown in the channel and collected on a transmission electron microscopy (TEM) grid for further structural investigations (see ESI for sample preparation). As shown in Figure 3c-d, the attachment of $\gamma-\mathrm{Fe}_{2} \mathrm{O}_{3}$ NPs on the surface of RITC-SiO ${ }_{2}$ NPs is evidenced. A surface coverage of $\approx 30 \%$ can be estimated from TEM images.

In summary, herein and for the first time, we presented a microfluidic platform for fast (all the process takes about 10 minutes) elaboration of magnetic and fluorescent nanohybrid assemblies based on manipulation of nanoliter microfluidic droplets loaded with nanoparticles and chemicals. This was achieved by using an automated microfluidic platform coupled with magnetic tweezers that allows sequential manipulation and different workflows on ferrofluid suspensions without leaving the microchannel. Our method is versatile; it can be applied to the surface coating of magnetic based NPs with any ligand and moreover to the assembly of different nanomaterials with magnetic NPs. In fact, here microfluidics is not exploited as a production technology, but as a synthesis investigation tool. We believe that magnetic tweezers technology combined with typical advantages of microfluidics could represent a very useful tool for screening chemical conditions saving time and reagents compared with standard chemical approach. However, to reach such a final goal it will be necessary to miniaturise and adapt different techniques known in colloidal chemistry such as light scattering methods in order to acquire fast data on the process and fast screening.

\section{Notes and references}

We thank Prof. J.C Bacri from MSC lab, Paris 7 for discussions. We thank Dr. Daniel Lévy and Aurélie Di Cicco for their help in TEM measurements. This work was supported in part by the Digidiag project (ANR) from the French government, the ARC foundation.

1 M.-C. Daniel and D. Astruc, Chem. Rev., 2004, 104, 293-346.

2 D. Ling, N. Lee and T. Hyeon, Acc. Chem. Res., 2015, 48, 12761285.

3 Hybrid Nanomaterials: Synthesis, Characterization, and Applications - Wiley Online Library, 2011.

4 Y. Song, J. Hormes and C. S. S. R. Kumar, Small, 2008, 4, 698711.

5 F. S. Majedi, M. M. Hasani-Sadrabadi, S. H. Emami, M. Taghipoor, E. Dashtimoghadam, A. Bertsch, H. Moaddel and P. Renaud, Chem. Commun. (Camb)., 2012, 48, 7744-6.

6 H. Song, D. L. Chen and R. F. Ismagilov, Angew. Chem. Int. Ed. Engl., 2006, 45, 7336-56.

7 S.-Y. Teh, R. Lin, L.-H. Hung and A. P. Lee, Lab Chip, 2008, 8, 198.

8 L. Frenz, A. El Harrak, M. Pauly, S. Bégin-Colin, A. D. Griffiths and J.-C. Baret, Angew. Chem. Int. Ed. Engl., 2008, 47, 6817-20.

9 M. Faustini, J. Kim, G.-Y. Jeong, J. Y. Kim, H. R. Moon, W.-S. Ahn and D.-P. Kim, J. Am. Chem. Soc., 2013, 135, 14619-26.

10 M. I. Bodnarchuk, L. Li, A. Fok, S. Nachtergaele, R. F. Ismagilov and D. V Talapin, J. Am. Chem. Soc., 2011, 133, 8956-60.

11 N. Hassan, V. Cabuil and A. Abou-Hassan, Angew. Chem. Int. Ed. Engl., 2013, 52, 1994-7.

12 C.-H. Yang, K.-S. Huang, Y.-S. Lin, K. Lu, C.-C. Tzeng, E.-C. Wang, C.-H. Lin, W.-Y. Hsu and J.-Y. Chang, Lab Chip, 2009, 9, 961-5.

13 A. Abou-Hassan, R. Bazzi and V. Cabuil, Angew. Chem. Int. Ed. Engl., 2009, 48, 7180-3.

14 A. Ito, M. Shinkai, H. Honda and T. Kobayashi, J. Biosci. Bioeng., 2005, 100, 1-11.

15 M. Chabert, K. D. Dorfman, P. de Cremoux, J. Roeraade and J.-L. Viovy, Anal. Chem., 2006, 78, 7722-7728.

16 C. N. Baroud, F. Gallaire and R. Dangla, Lab Chip, 2010, 10, 2032

17 A. Ali-Cherif, S. Begolo, S. Descroix, J.-L. Viovy and L. Malaquin, Angew. Chem. Int. Ed. Engl., 2012, 51, 10765-9.

18 B. Teste, A. Ali-Cherif, J. L. Viovy and L. Malaquin, Lab Chip, 2013, 13, 2344-9.

19 R. Massart, IEEE Trans. Magn., 1981, 17, 1247-1248.

20 R. Massart, E. Dubois, V. Cabuil and E. Hasmonay, J. Magn. Magn. Mater., 1995, 149, 1-5.

21 J.-P. Fortin, C. Wilhelm, J. Servais, C. Ménager, J.-C. Bacri and F. Gazeau, J. Am. Chem. Soc., 2007, 129, 2628-35.

22 C. Wu, J. Zheng, C. Huang, J. Lai, S. Li, C. Chen and Y. Zhao, Angew. Chemie, 2007, 119, 5489-5492. 